\title{
FREE QUOTIENTS OF FUNDAMENTAL GROUPS OF SMOOTH QUASI-PROJECTIVE VARIETIES
}

\author{
JOSE I. COGOLLUDO AND ANATOLY LIBGOBER
}

\begin{abstract}
We study the fundamental groups of the complements to curves on simply connected surfaces, admitting non-abelian free groups as their quotients. We show that given a subset of the Néron-Severi group of such a surface, there are only finitely many classes of equisingular isotopy of curves with irreducible components belonging to this subset for which the fundamental groups of the complement admit surjections onto a free group of a given sufficiently large rank. Examples of subsets of the Néron-Severi group are given with infinitely many isotopy classes of curves with irreducible components from such a subset and fundamental groups of the complements admitting surjections on a free group only of a small rank.
\end{abstract}

\section{Preface}

This note describes a result on the fundamental groups of the complements to curves $\mathcal{D} \subset V$ on a smooth simply connected projective surface $V$ such that $\pi_{1}(V \backslash \mathcal{D})$ admits a surjection on a free non-abelian group when the rank of free groups varies. To this end, we consider curves $\mathcal{D}$, with reduced components 1 , as elements of classes $\mathcal{C}(\Delta)$, parametrized by subsets $\Delta$ of the effective cone of $V$ in the sense that the classes of irreducible components of $\mathcal{D}$ are required to be in $\Delta$. An example of such a class is given by arrangements of lines in a projective plane: here $\Delta$ is the positive generator of $\mathrm{NS}\left(\mathbb{P}^{2}\right)$ and an arrangement of $n$ lines is viewed as a curve $\mathcal{D}$ of degree $n$. Grouping the curves by restricting the classes of irreducible components has a certain resemblance to the study of the distribution of the number fields with a fixed discriminant (cf. for example [5] or [1]).

In these circumstances, we show that for a fixed saturated (see Definition 1.1) set $\Delta$, there is a constant $M_{2}(\Delta)$ such that the existence of a surjection $\pi_{1}(V \backslash \mathcal{D}) \rightarrow F_{r}$ with $r>M_{2}(\Delta)$ implies that all components of $\mathcal{D}$ belong to a pencil of curves with the class of generic member being one of the classes in $\Delta$. In other words, the existence of a surjection of the fundamental group of a quasi-projective surface with compactification $V$ onto $F_{r}$ with a sufficiently large $r$ implies that the divisor at infinity $\mathcal{D}$ has a very special geometry (i.e., is a union of reducible curves which are members of a pencil or in other words $\mathcal{D}$ is composed of curves of a pencil).

The first named author is partially supported by PID2020-114750GB-C31 and Grupo "Álgebra y Geometría" of Gobierno de Aragón/Fondo Social Europeo. The second named author was partially supported by a grant from Simons Foundation.

${ }^{1}$ The topological space underlying the complement $V \backslash \mathcal{D}$ depends only on the support of $\mathcal{D}$ i.e. for a classification of the classes of fundamental groups of smooth quasi-projective varieties, without loss of generality one can assume that $\mathcal{D}$ is reduced. However, geometric detection of the fundamental groups, e.g. via study of the pencils (cf. proof of the Theorem 1.2 in Section 3, sometimes requires the consideration of non-reduced curves with the same support as $\mathcal{D}$. Below, it should be clear from the context when the consideration of non-reduced curves is necessary. 
This leads to the finiteness results for such classes of curves described below. We make a technical assumption on the singularities of $\mathcal{D}$ at intersections of different components, but not on the singularities of irreducible components outside of these intersections. This paper studies surjections $\pi_{1}(V \backslash \mathcal{D}) \rightarrow F_{r}$ which are essential in the sense that the images in $F_{r}$ of the elements of $\pi_{1}(V \backslash \mathcal{D})$ associated with each irreducible component of $\mathcal{D}$ (the meridians) are conjugates to the standard generators of $F_{r}$ (cf. condition $(* *)$ below for exact statement). We expect that a more careful analysis can show that the technical assumptions we made can be eliminated or substantially weakened ${ }^{2}$

Note that for arbitrary $r>1$ and any pencil $L$ on $V$, if one can considers a curve $\mathcal{D}=\bigcup_{i=1}^{r+1} D_{i}$ which is a union of $r+1$ members $D_{i}$ of $L$, then the rational dominant map onto $\mathbb{P}^{1}$ corresponding to $L$ induces the surjection $\pi_{1}(V \backslash \mathcal{D}) \rightarrow F_{r}$. Our result shows that given a set $\Delta$ of allowed classes in $\operatorname{NS}(V)$ of reduced components of $\mathcal{D}$, this is the only way to obtain curves admitting surjections of their fundamental groups of the complements onto $F_{r}$ satisfying $\left({ }^{* *}\right)$ with $r>M_{2}(\Delta)$. Hence, the study of fundamental groups with large free quotients depends on the study of the distribution of constants $M_{2}(\Delta)$. At this point we have explicit calculations of the constants $M_{2}(\Delta)$ for $V=\mathbb{P}^{2}$ and several other surfaces, provided in section 4 .

The main step in our argument is a statement about the pencils of curves on $V$ admitting sufficiently many fibers having as irreducible components only the curves with classes in Néron-Severi group in a chosen subset $\Delta$ of the effective cone. More precisely, we consider pencils $L \subset \mathbb{P}\left(H^{0}\left(V, \mathcal{O}_{V}(D)\right)\right)$ (i.e. $\left.\operatorname{dim} L=1\right)$ admitting $r+1$ distinct divisors $D_{1}, \ldots, D_{r+1}, D_{i} \in L$ whose classes of irreducible components of $D_{i}$ form a subset of $\Delta$ having cardinality $c \geq 1$. We show that the existence of such a pencil with $r>M_{2}(\Delta)$, implies that all elements of the pencil $L$ are already in a fixed class from $\Delta$. The claim about the free quotients of the fundamental groups follows immediately using [1] (cf. also [2]).

The above results admit a refinement. We also show that if $\pi_{1}(V \backslash \mathcal{D})$ admits a surjection onto $F_{r}$ then already for $r \geq 10$, such a curve $\mathcal{D}$ (having irreducible components in $\Delta$ ) must be a union of curves belonging to a pencil, with the generic member having Néron-Severi class in $\Delta$, with only finitely many exceptions depending on $\Delta$. The number of exceptions in general grows together with the set $\Delta$. For some $V$ and $\Delta \in \mathrm{NS}(V)$, the absence of surjections onto $F_{r}$ with only finitely many exceptions occurs already for some $r \leq 9$ (see section 5). However, for general $V$, $r \leq 9$ and certain $\Delta$ one expects infinitely many curves $\mathcal{D}$ with components in $\Delta$ admitting surjections onto $F_{r}$ and not being a union of members of a pencil with the class of a generic member in $\Delta$. It is an interesting problem to find the threshold $M_{1}(V, \Delta) \leq \min \left\{9, M_{2}(V, \Delta)\right\}$ such that for $r \leq M_{1}(V, \Delta)$ there are infinitely many fundamental groups of the complement to curves having $F_{r}$ as a quotient and irreducible components in $\Delta$, explicitly for a specific $(V, \Delta)$ with initial steps made in section 4 .

A precursor of such results is the following statement about arrangements of lines in $\mathbb{P}^{2}$ which was first shown in [10 and later improved in 6], 13. If an arrangement $\mathcal{A}$ of lines in $\mathbb{P}^{2}$, i.e an algebraic curve with all its components being in $\mathcal{C}([1])$ where $[1]$ is the positive generator of $\mathrm{NS}\left(\mathbb{P}^{2}\right)$, is such that there exist a pencil of curves of degree $d \geq 1$ admitting 5 or more members which are unions of

\footnotetext{
${ }^{2}$ maximal rank of a free quotient of a group sometimes is called corank. Here we consider essential surjections of groups which may have a corank greater than $r$.
} 
lines and such that $\mathcal{A}$ is the arrangement of lines belonging to these members of the pencil, then $d=1$ and therefore $\mathcal{A}$ is a central arrangement, i.e., is the union of lines all containing a fixed point (cf. section 4 for a discussion of this and other special cases). This implies that $\pi_{1}\left(\mathbb{P}^{2} \backslash \mathcal{A}\right)$ has no essential surjections onto $F_{r}, r \geq 4$ except for the central arrangements. On the other hand there are infinitely many non-central arrangements of lines with $\pi_{1}\left(\mathbb{P}^{2} \backslash \mathcal{A}\right)$ having essential surjections onto $F_{2}$. In the language introduced in this paper $M_{2}\left(\mathbb{P}^{2},[1]\right)=3$ and where $\Delta=[1]$ and $[d] \in \mathrm{NS}\left(\mathbb{P}^{2}\right)$ is the class of a curve of degree $d$.

The sets $\Delta$, used below, must satisfy certain consistency conditions.

Definition 1.1. Let $\operatorname{Eff}(V) \subset \mathrm{NS}(V)$ denote the effective cone of $V$ (cf. 9]). We call a subset $\Delta \subset \operatorname{Eff}(V)$ saturated if the following condition is satisfied: if a class $d \in \Delta$ admits a split $d=d_{1}+d_{2}, d_{1}, d_{2} \in \operatorname{Eff}(V)$ then both classes $d_{1}$ and $d_{2}$ are in $\Delta$.

This condition assures that if an "allowed" (i.e. from $\Delta$ ) class in $\operatorname{NS}(V)$ is represented by a reducible or non-reduced curve, then its irreducible and reduced components are allowed as well. Other conditions of being saturated may lead to different implications on the ranks of free quotients. We work with finite saturated sets. Note that a minimal saturated subset containing a finite set is finite (cf. Lemma 3.1.

Since we are interested in $\pi_{1}(V \backslash \mathcal{D})$, we consider curves $\mathcal{D}$ on smooth surfaces up to the following equivalence relation: two curves $\mathcal{D}^{\prime}, \mathcal{D}^{\prime \prime}$ are equivalent if there is a topologically equisingular deformation of $\mathcal{D}^{\prime}$ into $\mathcal{D}^{\prime \prime}$. Such deformations do not alter the fundamental groups of the complements and all the finiteness statements are made for such equivalence classes.

We also say that a curve $\mathcal{D}$ is composed of curves of a pencil ${ }^{3}$ in a linear system $H^{0}(V, \mathcal{O}(D))$ if there is a partition of irreducible components of $\mathcal{D}$ into groups such that the union, possibly with multiplicities, of irreducible components in each group is one of the curves in the pencil.

The results in this paper use the following assumptions:

(*) All singular points of $\mathcal{D}$ belonging to more than one component are ordinary multiple points of the multiplicity equal to the number of components containing this singular point i.e. locally are transversal intersection of smooth germs.

$\left.{ }^{* *}\right)$ The surjections $\pi_{1}(V \backslash \mathcal{D}) \rightarrow F_{r}$ we consider are essential i.e. take each meridian of a component of $\mathcal{D} 4$ to an element in a conjugacy class of either one of $r+1$ generators of $F_{r}=\left\langle x_{1}, \ldots, x_{r+1}: x_{1} \cdot \ldots \cdot x_{r+1}=1\right\rangle$.

With these conventions, we have the following:

Theorem 1.2. Let $V$ be a smooth simply connected projective surface. Let $\Delta \subset$ $\mathrm{NS}(V)$ be a saturated subset of the effective cone and let $\mathcal{D}$ be a reduced curve having classes of its irreducible components in $\Delta$ and satisfying condition $\left({ }^{*}\right)$. Then there is a constant $M_{2}(V, \Delta)$ such that if $\pi_{1}(V \backslash \mathcal{D})$ admits an essential free quotient (i.e. for which the condition $\left({ }^{* *}\right)$ is satisfied) with $r>M_{2}(V, \Delta)$ then $\mathcal{D}$ is composed of curves in a pencil with the property that the class of its generic element in $\operatorname{NS}(V)$ is a class $\delta \in \Delta$.

\footnotetext{
${ }^{3}$ In footnote [20], p.214, Zariski describes such a curve as "a curve made up of curves belonging to one and the same pencil".

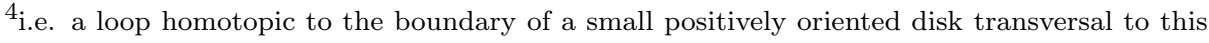
component at a smooth point.
} 
Moreover, there is a constant $M_{1}(V, \Delta)<10$ such that for $r>M_{1}(V, \Delta)$ there is only finite number $N(V, r, \Delta)$ of isotopy classes of curves $\mathcal{D}$ with components from $\Delta$ and admitting a surjection $\pi_{1}(V \backslash \mathcal{D}) \rightarrow F_{r}$ but not composed of a pencil in $H^{0}(V, \mathcal{O}(D))$ where $D$ is a divisor in a class $\delta \in \Delta$.

The implication of this result on the structure of quasi-projective groups which are the fundamental groups of the complements to curves on a given surface with given classes of components is as follows:

Corollary 1.3. Given a saturated set $\Delta$ of classes in $\mathrm{NS}(V)$ consider the following trichotomy for the curves as in Theorem 1.2 i.e. with classes of irreducible components in $\Delta$, satisfying conditions $\left({ }^{*}\right)$ and having a free essential quotient (i.e. satisfying (**)) of a fixed rank $r>1$.

(A) There exist infinitely many isotopy classes of curves admitting an essential surjections $\pi_{1}(V \backslash \mathcal{D}) \rightarrow F_{r}$.

(B) There are finitely many isotopy classes of curves $\mathcal{D}$ admitting essential surjections $\pi_{1}(V \backslash \mathcal{D}) \rightarrow F_{r}, r>1$.

(C) $\mathcal{D}$ admitting an essential surjection $\pi_{1}(V \backslash \mathcal{D}) \rightarrow F_{r}$, is composed of curves of a pencil having generic member in $\Delta$. There are finitely many isotopy classes of such $\mathcal{D}$ for given $\Delta$.

All three cases are realizable at least for some $(V, \Delta)$. Case (B) takes place for $r \geq 10$ for any $\Delta$. There exists a constant $M_{2}(V, \Delta)$ such that for $r>M_{2}(V, \Delta)$ one has case (C). In the latter case, $\pi_{1}(V \backslash \mathcal{D})$ splits as an amalgamated product $H *_{\pi_{1}(\Sigma)} G$ where $\Sigma$ is a Riemann surface which is a smooth member of the pencil, $H$ is coming from a finite set of groups associated with the linear system $H^{0}(V, \mathcal{O}(D))$, $D$ is a divisor having class $\delta \in \Delta$ and $G$ is an extension:

$$
0 \rightarrow \pi_{1}(\Sigma) \rightarrow G \rightarrow F_{r} \rightarrow 0
$$

Note that the actual values of $r$ for which one has each of the cases $\sqrt{A},(B)$ or (C), varies for specific surfaces, see section 4 .

The fundamental groups as in Theorem 1.2 admit a geometric surjections onto a free group i.e. which are induced by holomorphic maps onto punctured $\mathbb{P}^{1}$ (cf. proof of Theorem 1.2). In fact, Theorem 1.2 is deduced from the following result on the reducible fibers of primitive pencils of curves 5

Theorem 1.4. Let $V, \Delta$ be as in Theorem 1.2. Then there is a constant $M_{2}(V, \Delta)$ such that for $r>M_{2}(V, \Delta)$, any primitive pencil of curves on $V$ such that:

(i) it has $r+1$ members whose classes of its irreducible components are in $\Delta$,

(ii) all components of each of these $r+1$ members are reduced,

(iii) the generic members of the pencil intersecting transversally at any base point have an element in $\Delta$ as a class.

Moreover, there exists a constant $M_{1}(V, \Delta)$ such that $2 \leq M_{1}(V, \Delta) \leq \min \left\{9, M_{2}(V, \Delta)\right\}$ and such that for $r>M_{1}(V, \Delta)$ there are only finitely many types of primitive pencils satisfying (ii), (ii) and (iii).

Theorems 1.2 and 1.4 are proven in section 3 where we also give the definitions of the classes of curves we consider and where we define the thresholds $M_{i}(V, \Delta), i=1,2, N(V, r, \Delta)$ and $K(V, \Delta)$ controlling the the fundamental groups

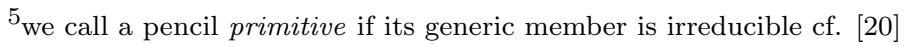


of the complements to curves for various ranks of their free quotients. One of the steps in the proof of Theorem 1.4 (cf. 13 ) is the following inequality, which may have an independent interest:

Corollary 1.5. Let $r+1$ be the number of reducible and reduced members in a pencil in a linear system $\mathbb{P}\left(H^{0}\left(V, \mathcal{O}_{V}(D)\right)\right)$, having classes of irreducible components in a saturated set $\Delta$ and for which the union of components of these $r+1$ members satisfies condition $(*)$. Then

$$
r+1 \leq 2 \frac{e(V)+3 D^{2}+2 K D}{D^{2}+K D+\min \left(\sum e\left(F_{j}\right)\right)}
$$

where the sum is taken over all irreducible components $F_{j}$ of a member of the pencil and the minimum is taken over these $r+1$ members of the pencil.

The rest of the paper deals with examples and the values of the thresholds $M_{i}(V, \Delta), N(V, r, \Delta)$ and $K(V, \Delta)$. In section 4 we discuss the free quotients for the curves on $\mathbb{P}^{2}$ in the classes $\mathcal{C}(\Delta)$ where $\Delta=\{[1], \ldots,[k]\}$ i.e. with irreducible components having degrees not exceeding $k$, and section 5 deals with examples on more general surfaces. More detailed estimates for the thresholds introduced here will be given elsewhere. The proofs are based on the estimates of the topological Euler characteristic of fibers of pencils in terms of Euler characteristics of components of degenerate fibers but depends also on asymptotic of ranks of cohomology of line bundles corresponding to nef divisors (cf. [9]).

Finally, we want to thank anonymous referee for careful reading of the manuscript and very useful comments. The first named author wants to thank the Fulbright Program and the Spanish Ministry of Ciencia, Innovación y Universidades for the grant to collaborate at UIC with the second named author.

\section{Summary of SOME NOtations}

$\Delta$ is a saturated subset of $\operatorname{NS}(V)$ where $V$ is a smooth projective simplyconnected surface.

$M_{2}(V, \Delta) \in \mathbb{Z}_{+}$(or simply $M_{2}(\Delta)$; the same with similar notations below) is the threshold for the ranks of free groups $F_{r}$ such that beyond it (i.e. for $r>M_{2}(V, \Delta)$ ) existence of surjection onto free group of $\pi_{1}(V \backslash \mathcal{D})$, where $\mathcal{D}$ is a curve with classes of irreducible components in $\Delta$, implies that $\mathcal{D}$ is a union of curves beloning to a pencil in complete linear system of a curve with class in $\Delta$ (cf. end of the proof of the theorem 1.4).

$M_{1}(V, \Delta) \in \mathbb{Z}_{+}$is the threshold for the ranks of free groups $F_{r}$ above which (i.e. for $r>M_{1}(V, \Delta)$ ) there are only finitely many isotopy classes of curves with fundamental groups of the complements admitting surjection onto $F_{r}$ (cf. Theorems 1.2 and 1.4 .

$N(V, r, \Delta) \in \mathbb{Z}_{+}$is the number of curves $\mathcal{D}$ with irreducible components having classes in a saturated set $\Delta$, which admit a surjection onto a free group $F_{r}, r>$ $M_{1}(V, \Delta)$ (cf. theorem 1.2 ).

$K(V, \Delta)$ is a positive integer such that for pencils in $H^{0}(V, \mathcal{O}(D))$ where $D$ is such that for any $\delta \in \Delta$ one has $D-K(V, \Delta) \delta \in \operatorname{Eff}(V)$, there are at most 12 reducible fibers with all irreducible components on $\Delta$ (cf. Corollary 3.3). 
$M_{k}=M_{2}\left(\mathbb{P}^{2}, \Delta_{k}\right)$ where $\Delta_{k}$ is the collection of classes of $\mathcal{O}_{\mathbb{P}^{2}}(1), \ldots, \mathcal{O}_{\mathbb{P}^{2}}(k)$ (cf. section 4).

\section{Proof of the Main theorem}

In this paper, only finite saturated subsets of the effective cone are considered. The following result verifies that the finiteness condition is preserved when considering the saturated subset spanned by a finite family of divisor classes.

Lemma 3.1. The saturated subset spanned by a finite subset of $\mathrm{Eff}(V)$ is finite.

Proof. Let $H$ be an ample divisor. For $d_{1}, \ldots, d_{s}, d_{i} \in \operatorname{Eff}(V)$, let $\Delta\left(d_{1}, \ldots, d_{s}\right)$ be the intersection of all saturated subsets containing $d_{1}, \ldots, d_{s}$. For any $\delta \in \Delta(d), d \in$ $\operatorname{Eff}(V)$ one has $(\delta, H) \leq(d, H)$. Therefore $\Delta(d)$ is a discrete subset of a compact set $\{S \in \overline{\operatorname{Eff}(V)} \otimes \mathbb{R} \mid(S, H) \leq(d, H)\}$ (since, as follows from the Hodge index theorem, $\operatorname{Eff}(V) \otimes \mathbb{R}$ is a cone over a compact set). Hence it is finite. Alternatively, the claim can be derived from 3 Theorem 4.10b. Finally, if $d_{1}, \ldots, d_{s}$ is a finite subset of $\operatorname{Eff}(V)$ then since for their span one has $\Delta\left(d_{1}, . ., d_{s}\right)=\bigcup \Delta\left(d_{i}\right)$ and hence it is finite as well.

The following will be used to obtain estimates of the number of members of pencils on a surface $V$ having classes of irreducible components in a fixed saturated subset $\Delta$.

Proposition 3.2. Let $\Delta$ be a saturated subset of $\operatorname{Eff}(V)$ and $s=\operatorname{Card} \Delta$. Let $\mathcal{F}$ be a curve with irreducible components $F_{j}, j=1, \ldots, \mathcal{J}, \mathcal{J}>1$ which moves in a pencil such that mutiplicity of each component of $\mathcal{F}$ in this pencil is 16 . Let $[\mathcal{F}]=D=\sum_{i=1}^{s} m_{i} d_{i}$ be the class of $\mathcal{F}$ in the free abelian group generated by $d_{i} \in \Delta \subset \mathrm{NS}(V)$. For all $\alpha>\frac{5}{3}$ and all but finitely many $\left(m_{1}, \ldots, m_{s}\right) \in \mathbb{Z}_{\geq 0}^{s}$ one has

$$
\frac{\frac{e(V)}{3}+D^{2}+\frac{2}{3} K D}{D^{2}+\sum_{j=1}^{\mathcal{J}} e\left(F_{j}\right)+K D}<\alpha
$$

$\left(e\left(F_{j}\right)\right.$ denotes the topological Euler characteristic of the component $\left.F_{j}\right)$.

Proof. Firstly, observe that the assumptions of Proposition imply that the denominator in (2) is non-zero. Otherwise, the equality given by adjunction formula for $\mathcal{F}$ holds with no corrections and hence $\mathcal{F}$ must be a smooth curve. Since we assume that $\mathcal{J}>1$, the curve $\mathcal{F}$ is disconnected, and by Zariski connectedness theorem, generic fiber in the pencil in which $\mathcal{F}$ moves must be disconnected. In this case, the element of the pencil corresponding to the branch point of the covering of $\mathbb{P}^{1}$ given by Stein factorization must be non-reduced which contradicts our assumption.

Next note that one has

$$
e\left(F_{j}\right)=-K F_{j}-F_{j}^{2}+\sum_{P \in \operatorname{Sing}\left(F_{j}\right)}\left(2 \delta\left(F_{j}, P\right)-b\left(F_{j}, P\right)+1\right)
$$

where $P$ runs through the set $\operatorname{Sing}\left(F_{j}\right)$ of singular points of the curve $F_{j}$ and $\delta\left(F_{j}, P\right), b\left(F_{j}, P\right)$ are respectively the $\delta$ invariant and the number of branches of $F_{j}$

\footnotetext{
$6_{\text {i.e. all components }} F_{j}$ of this particular element of the pencil are reduced; there is no restrictions on multiplicities of components of other members of the pencil.
} 
at $P$ (cf. [16]). Each summand in summation in (3) is non-negative. Hence the denominator in (2) satisfies:

$$
D^{2}+\sum_{j=1}^{\mathfrak{J}} e\left(F_{j}\right)+K D \geq D^{2}-\sum m_{i} K d_{i}-m_{i} d_{i}^{2}+K D=D^{2}-\sum m_{i} d_{i}^{2} .
$$

Since we assume that the pencil consists of only reduced members, in the decomposition $D=\sum_{i, d_{i}^{2} \geq 0} m_{i} d_{i}+\sum_{i, d_{i}^{2}<0} m_{i} d_{i}$, for coefficients of $d_{i}$ for which $d_{i}^{2}<0$, one has $m_{i}=1$. Indeed, two numerically equivalent irreducible curves with negative self-intersection which appears in a reducible member $\mathcal{F}$ of the pencil more than once and must coincide, since they cannot be deformations of each other. Therefore $D^{2}-\sum m_{i} d_{i}^{2}=\sum\left(m_{i}^{2}-m_{i}\right) d_{i}^{2}+2 \sum m_{i} m_{j} d_{i} d_{j}>0$. and hence the inequality 2 would follow from

$$
(\alpha-1) D^{2}-\alpha \sum m_{i} d_{i}^{2}-\frac{2}{3} K D>\frac{e(V)}{3} .
$$

Indeed, from (4) and (5) one has

$$
\alpha\left(D^{2}+\sum_{j=1}^{J} e\left(F_{j}\right)+K D\right) \geq \alpha\left(D^{2}-\sum m_{i} d_{i}^{2}\right)>D^{2}+\frac{2}{3} K D+\frac{e(V)}{3} .
$$

To show (5), let $D=\sum m_{i} d_{i}, d_{i} \in \Delta$ be a divisor which is a reducible member of a pencil (i.e. $\operatorname{dim} H^{0}(V, \mathcal{O}(D)) \geq 2$ ). By Riemann-Roch

$$
-D K=2\left(\operatorname{dim} H^{0}(\mathcal{O}(D))-\operatorname{dim} H^{1}(\mathcal{O}(D))+\operatorname{dim} H^{2}(\mathcal{O}(D))-2 \chi(V)-D^{2} .\right.
$$

The asymptotic behavior of the cohomology of nef divisors (cf. the proof of Theorem 1.4.40 9 p.69 or 8) implies that for all but finitely many $\left(m_{1}, \ldots, m_{s}\right)$, i.e. those in the compact subset of $\overline{\operatorname{Eff}(V)} \subset \mathrm{NS}(V) \otimes \mathbb{R}$ where $\operatorname{dim} H^{1}(\mathcal{O}(D))$ exceeds the dimension of $H^{0}(\mathcal{O}(D))$, one has $\chi(\mathcal{O}(D)) \geq 0$. For those $\left(m_{1}, \ldots, m_{s}\right)$,

$$
-D K \geq-2 \chi(V)-D^{2} \text {. }
$$

Hence for all but finitely many $\left(m_{1}, \ldots, m_{s}\right) \in \mathbb{Z}_{>}^{s}$ we have the following inequality for the left hand side of (5):

$$
(\alpha-1) D^{2}-\alpha \sum m_{i} d_{i}^{2}-\frac{2}{3} K D \geq\left(\alpha-\frac{5}{3}\right) D^{2}-\alpha \sum m_{i} d_{i}^{2}-\frac{4}{3} \chi(V) .
$$

It only remains to show that if $\alpha>\frac{5}{3}$ then for all but finitely many $m_{i}$ one has

$$
\left(\alpha-\frac{5}{3}\right) D^{2}-\alpha \sum m_{i} d_{i}^{2}>\frac{e(V)}{3}+\frac{4}{3} \chi(V) .
$$

To see (8) note that, as was already mentioned, in the decomposition $D=\sum_{i, d_{i}^{2} \geq 0} m_{i} d_{i}+$ $\sum_{i, d_{i}^{2}<0} m_{i} d_{i}$, since we assume that the pencil consists of only reduced members, for $d_{i}$ with $d_{i}^{2}<0$ one has $m_{i}=1$. Hence for left hand side of $(8)$ one has

$$
\begin{gathered}
\left(\alpha-\frac{5}{3}\right) D^{2}-\alpha \sum m_{i} d_{i}^{2}= \\
\alpha \sum_{i, d_{i}^{2} \geq 0}\left(m_{i}^{2}-m_{i}\right) d_{i}^{2}-\frac{5}{3} \sum_{i} m_{i}^{2} d_{i}^{2}+2\left(\alpha-\frac{5}{3}\right) \sum_{i<j} m_{i} m_{j} d_{i} d_{j} \geq \\
\left(\alpha-\frac{5}{3}\right) \sum_{i, d_{i}^{2} \geq 0} m_{i}^{2} d_{i}^{2}-\alpha \sum_{i, d_{i}^{2}>0} m_{i} d_{i}^{2}+2\left(\alpha-\frac{5}{3}\right) \sum_{i<j} m_{i} m_{j} d_{i} d_{j} \geq
\end{gathered}
$$




$$
2\left(\alpha-\frac{5}{3}\right) \sum_{i<j} m_{i} m_{j} d_{i} d_{j}
$$

since for $\alpha>\frac{5}{3}$ in the left hand side of the last inequality the first two terms are either zero (if $d_{i}^{2}=0$ for all $i$ ) or give a quadratic function in $m_{i} \geq 0$ with the terms of degree 2 representing a positive definite diagonal quadratic form (in variables $\left.m_{i}\right)$. Hence one has the last inequality for all $\left(m_{1}, \ldots, m_{s}\right) \in \mathbb{Z}_{\geq}^{s}$ but a finite set since exceptions are given by solutions of the opposite inequality which belong to a compact subset of $\left(\mathbb{R}_{\geq}\right)^{s}$. Finally

$$
2\left(\alpha-\frac{5}{3}\right) \sum_{i<j} m_{i} m_{j} d_{i} d_{j}>\frac{e(V)}{3}+\frac{4}{3} \chi(V)
$$

for $\alpha>\frac{5}{3}$ since, as was shown earlier, $\mathcal{F}$ contains intersecting irreducible components. This shows 8 for all but finitely many $\left(m_{1}, \ldots, m_{s}\right)$ and hence the result follows.

Proof of Theorem 1.4. Let $B \subset V$ be the base locus of a pencil satisfying conditions (ii),(ii), (iii). Assumption (iii) implies that the pencil is free of fixed components, the rational map corresponding to the pencil extends to a regular map $\Phi: \tilde{V} \rightarrow \mathbb{P}^{1}$ on the blow up $\tilde{V}$ of $V$ at $B$ and that $\operatorname{Card} B=\left(\Phi^{-1}(b) \cdot \Phi^{-1}(b)\right), b \in \mathbb{P}^{1}$. Let $B^{\prime} \subset \mathbb{P}^{1}$ the set of critical values of $\Phi$ and let $R \subset B^{\prime}$ be the subset corresponding to the $r+1$ members satsifying the conditions (ii)-(iii) of the statement of the Theorem. For $b^{\prime} \in B^{\prime}$ and any $p \in \mathbb{P}^{1} \backslash B^{\prime}$, let $e_{\text {rel }}\left(b^{\prime}\right)=e\left(\Phi^{-1}\left(b^{\prime}\right)\right)-e\left(\Phi^{-1}(p)\right)$ be the relative Euler characteristic of the fiber at $b^{\prime}$ (this is independent of $p$ ). It follows from the additivity of the Euler characteristic (cf. also [7]) that

$$
e(V)+\operatorname{Card} B=2 e\left(\Phi^{-1}(p)\right)+\sum_{b^{\prime} \in B^{\prime}} e_{r e l}\left(b^{\prime}\right)
$$

where $p \in \mathbb{P}^{1} \backslash B^{\prime}$. It follows by the adjunction applied on $V$, that for $p \in \mathbb{P}^{1} \backslash B^{\prime}$ one has $e\left(\Phi^{-1}(p)\right)=-\left(K D+D^{2}\right)$ where $D \subset V$ is the class of any fiber of $\Phi$. Note that for any $b^{\prime} \in B^{\prime}$ one has $e_{\text {rel }}\left(b^{\prime}\right) \geq 0$ (cf. [7] or [17, Ch.4, Theorem 6 and 7) and, as was mentioned, Card $B=D^{2}$. Therefore

$$
\begin{aligned}
e(V)+D^{2} & =-2\left(K D+D^{2}\right)+\sum_{b^{\prime} \in R} e_{r e l}\left(b^{\prime}\right)+\sum_{b^{\prime} \in B^{\prime} \backslash R} e_{r e l}\left(b^{\prime}\right) \\
& \geq-2\left(K D+D^{2}\right)+\sum_{b^{\prime} \in R} e_{r e l}\left(b^{\prime}\right)
\end{aligned}
$$

For any $b^{\prime} \in R$, such that the class of fiber $\Phi^{-1}\left(b^{\prime}\right)$ is $D=\sum m_{i} d_{i}$, using as a lower bound for the Euler characteristic of a reducible curve on a surface, the expression for the Euler characteristic in the case when all irreducible components are smooth and intersect transversally at distinct points, one has

$$
e_{r e l}\left(b^{\prime}\right) \geq \sum-m_{i} d_{i}\left(K+d_{i}\right)-\sum \frac{m_{i}\left(m_{i}-1\right)}{2} d_{i}^{2}-\sum_{i<j} m_{i} m_{j} d_{i} d_{j}+D(K+D) .
$$

Here the first term represents the sum of Euler characteristics of smooth irreducible components, the second is the count of intersection points among the $m_{i}$ curves in each class $d_{i}$, the third term is the number of intersection points among curves in classes $d_{i}, d_{j}, i \neq j$ and last term is negative of the Euler characteristic of the 
smooth fiber of $\Phi$. Replacing $D$ by $\sum m_{i} d_{i}$ we obtain by (11) that $e_{r e l}\left(b^{\prime}\right), b^{\prime} \in R$ is greater than or equal to:

$$
\begin{gathered}
-\sum m_{i} K d_{i}+\sum d_{i}^{2}\left(-m_{i}-\frac{m_{i}\left(m_{i}-1\right)}{2}+m_{i}^{2}\right)+\sum_{i<j} d_{i} d_{j}\left(2 m_{i} m_{j}-m_{i} m_{j}\right)+\sum m_{i} K d_{i} \\
=\sum d_{i}^{2} \frac{m_{i}\left(m_{i}-1\right)}{2}+\sum_{i<j} d_{i} d_{j} m_{i} m_{j}= \\
\frac{D^{2}-\sum m_{i}\left(d_{i}^{2}\right)}{2}=\frac{D^{2}+K D+\sum_{F_{j} \in \Phi^{-1}\left(b^{\prime}\right)} e\left(F_{j}\right)}{2} .
\end{gathered}
$$

Selecting $b^{\prime} \in R$ for which $\frac{D^{2}-\sum m_{i}\left(d_{i}^{2}\right)}{2}$ is the smallest $\Delta$, one obtains

$$
e(V)+D^{2} \geq-2\left(K D+D^{2}\right)+(r+1) \frac{D^{2}+K D+\sum_{F_{j} \in \Phi^{-1}\left(b^{\prime}\right)} e\left(F_{j}\right)}{2} .
$$

Hence the last inequality implies that for $\alpha$ as the Proposition 3.2 one has:

$$
r+1 \leq 2 \frac{e(V)+3 D^{2}+2 K D}{D^{2}+K D+\sum e\left(F_{j}\right)}<6 \alpha .
$$

From Proposition 3.2 , for $\alpha>\frac{5}{3}$ we obtain that, with only finitely many exceptions $\Xi=\left\{D \mid D=\sum m_{i} D_{i}, D \notin \Delta\right\},{ }^{7}$ a pencil in the linear system $H^{0}(V, \mathcal{O}(D))$ will have no more than 10 reducible fibers with all components in $\Delta$. Hence if $r$ is such that there are infinitely many pencils having $r+1$ reducible fibers with components in $\Delta$ then $r+1 \leq 10$. Denoting by $M_{1}(V, \Delta)$ the largest $r$ such that there are infinitely many pencils with $r+1 \geq 3$ members having irreducible components in $\Delta$, we see that $2 \leq M_{1}(V, \Delta) \leq 9$.

Since each linear system may have only a finitely many isotopy classes of pencils (it is bounded by the number of strata in a stratification of the set of pair $(l, D i s c)$ where $l$ is a line and Disc is the discriminant in the complete linear system $\mathbb{P}\left(H^{0}(\mathcal{O}(D))\right)$, this may create only a finite set of pencils in $H^{0}(\mathcal{O}(D))$ with $D \in \Xi$ whose classes of irreducible components are in $\Delta$ for $r+1>10$ of its members. If $M_{2}(V, \Delta)+1$ is the maximal number of reducible members in this finite set of pencils, then for $r>M_{2}(V, \Delta)$ the class of members of the pencil will be in $\Delta$. This shows the theorem.

Proof of theorem 1.2. Note that existence of a surjection $\pi_{1}(V \backslash \mathcal{D}) \rightarrow F_{r}, r \geq 2$, by work [1, implies that there is a surjective holomorphic map with connected fibers $V \backslash \mathcal{D} \rightarrow C \backslash R$, where $C$ is a smooth curve and $R \subset C$ is a finite set such that $\operatorname{rk} H^{1}(C \backslash R) \geq r+18^{8}$ Indeed, for any $\chi \in C h a r F_{r}$ the lower degree terms sequence corresponding to the Hochschild-Serre spectral sequence

$$
H^{p}\left(F_{r}, H^{q}(K, \mathbb{C})\right) \Rightarrow H^{p+q}\left(\pi_{1}(V \backslash \mathcal{D}, \tilde{\chi})\right.
$$

of the extension $0 \rightarrow K \rightarrow \pi_{1}(V \backslash \mathcal{D}) \rightarrow F_{r} \rightarrow 0$, where $\tilde{\chi}$ is the pullback of the character $\chi$ to the character of $\pi_{1}(V \backslash \mathcal{D})$, implies that $0 \rightarrow H^{1}\left(F_{r}, \chi\right) \rightarrow$ $H^{1}\left(\pi_{1}(V \backslash \mathcal{D}, \tilde{\chi})\right.$. Since $\operatorname{dimChar} F_{r}=r$ and $\operatorname{rk} H^{1}\left(F_{r}, \chi\right) \neq 0(\chi \neq 1, r>1)$, it follows that a surjection onto $F_{r}$ belongs to an irreducible component $\Sigma, \operatorname{dim} \Sigma \geq r$ of the characteristic variety containing the torus of the characters $\tilde{\chi}$ of $\pi_{1}(V \backslash \mathcal{D})$

\footnotetext{
$7_{\text {i.e. }}$ the set of linear systems in the semigroup generated by classes in $\Delta$ which are outside of $\Delta$.

${ }^{8}$ condition $(* *)$, i.e. that surjection is essential, is used later in the proof.
} 
with non-vanishing cohomology. The component $\Sigma$ is the pullback of the torus Char $H_{1}(C \backslash R)$ via an admissible map $V \backslash \mathcal{D} \rightarrow C \backslash R$ of the Theorem 1.6 of [1.

This map extends to a map having indeterminacy points at a subset of $V$ of codimension two. More specifically, the map is well defined outside of a finite subset $B \subset V$ which is a subset of the set of intersections of components of $\mathcal{D}$ i.e. subset of the union of $D^{\prime} \cap D^{\prime \prime}$ where $D^{\prime}, D^{\prime \prime}$ run through the set of pairs of irreducible components of $\mathcal{D}$. Moreover, since we assume $\left(\right.$ cf. $\left(^{*}\right)$ ) that the intersections of the components of $\mathcal{D}$ are transversal, this map extends to a holomorphic map $\Phi: \tilde{V} \rightarrow C$ of the single blow up of $V$ at each of the indeterminacy points on $V$. The generic fiber of this map is irreducible since the map $V \backslash \mathcal{D} \rightarrow C \backslash R$ is admissible. One has $\pi_{1}(\tilde{V}) \rightarrow \pi_{1}(C)$ and since we assume $\pi_{1}(V)=0$ this shows that $C=\mathbb{P}^{1}$ and Card $R \geq r+1$. Moreover, $\Phi^{-1}(R) \subset \tilde{V}$ can be identified with $\mathcal{D}$ since due to our assumption on the intersection of the components on $V$ no new components introduced as result of elimination of base points of the pencil and since component of $\mathcal{D}$ satisfy condition $(*)$. In particular, this pencil satisfies conditions (ii),(iii) of Theorem 1.4 for Card $R$ of its fibers. The condition (ii) for this pencil, i.e. that the fibers over $R$ are reduced ${ }^{9}$ follows from $\left.{ }^{* *}\right)$. Indeed, restriction of the map $\Phi: \tilde{V} \rightarrow \mathbb{P}^{1}$ on a small disk transversal to component $D_{i}$ of the fiber $\sum m_{i} D_{i}$ of the pencil is given by $z \rightarrow z^{m_{i}}$. Hence the corresponding map of the fundamental groups $\pi_{1}(V \backslash \mathcal{D}) \rightarrow \pi_{1}\left(\mathbb{P}^{1} \backslash R\right)$ takes meridian of the component to a conjugate of $x_{s}^{m_{i}}$ where $x_{s}$ is generator of the fundamental group of the complement in $\mathbb{P}^{1}$ running around the point corresponding to the fiber $\sum m_{i} D_{i}$. Since the sequence of inclusions Char $F_{r} \subset \Sigma=\Phi^{*}\left(\operatorname{CharH}_{1}\left(\mathbb{P}^{1} \backslash R\right)\right) \subset$ Char $\pi_{1}(V \backslash \mathcal{D})$ is dual to composition $H_{1}(V \backslash \mathcal{D}, \mathbb{Z}) \rightarrow H_{1}\left(\mathbb{P}^{1} \backslash R, \mathbb{Z}\right) \rightarrow F_{r} / F_{r}^{\prime}$ and composition of these map by assumption $\left({ }^{*}\right)$ of the Theorem 1.2 takes a meridian of a component of $\mathcal{D}$ to an indivisible element in $F_{r} / F_{r}^{\prime}$, so is the case for the map $H_{1}(V \backslash \mathcal{D}) \rightarrow H_{1}\left(\mathbb{P}^{1} \backslash R\right)$ and hence all components of $\mathcal{D}$, considered as components of members of the pencil $\Phi$ are reduced and (ii) is satisfied. Now the Theorem 1.2 is an immediate consequence of Theorem 1.4

The inequalities considered in the proof of the Theorem 1.4 have the following consequence, giving under special assumptions, a replacement of the part of conclusion of theorem 1.4 stipulating possibility of "finitely many exception".

Corollary 3.3. Let $\Delta \subset \mathrm{NS}(V)$ denote a saturated subset. Assume that either $\Delta$ contains a class $d$ such that $d^{2}>0$ or that one has $d^{2}<0, K d \leq 0$ for all classes in $\Delta$. Then there exists a smallest constant $K(V, \Delta) \in \mathbb{Z}_{+}$such that for a pencil of curves having class $D \in \mathrm{NS}(V)$ satisfying the inequality $D>d{ }^{10}$ for all $d \in K(V, \Delta) \Delta$ where

$$
K(V, \Delta) \Delta=\left\{d \in \mathrm{NS}(V) \mid d=\sum m_{i} d_{i}, d_{i} \in \Delta, m_{i}>K(V, \Delta)\right\}
$$

the number $r(V)+1$ of reducible fibers with components in $\Delta$ is 11 or less.

Remark 3.4. Recall that we are considering only the pencils subject to condition on meridians stated in the theorem 1.2. It excludes the pencils with all components of reduced fibers being in $\Delta$ having only classes $d$ with $d^{2}<0$ in

\footnotetext{
${ }^{9}$ outside of a finite subset $B^{\prime} \subset \mathbb{P}^{1}$ the smooth fibers of the holomorphic map $\Phi$ are diffeomorphic and one has the inclusion $R \subseteq B^{\prime}$. This pencil, of course, may have reducible fibers over points in $C \backslash R$ and its components do not need to have classes in $\Delta$.

10i.e. $D-d \in \operatorname{Eff}(V)$.
} 
$D=\sum m_{i} d_{i}, m_{i}>1$. Proposition 5.12 below shows that there are pencils having arbitrary large number of reducible components with negative self-intersections and positive intersection with canonical class (albeit on different surfaces).

Remark 3.5. In the case $(V, \Delta)=\left(\mathbb{P}^{2},[1], \ldots,[k]\right)$ the constant $K(V, \Delta)$ represents the threshold for the degrees of pencils having "large number" of members with degrees of components at most $k$. The "large" means more than 12 but it is smaller for small $k$ (cf. section 4).

Proof of Corollary 3.3. Consider the inequality (5) for $\alpha=2$ i.e.

$$
D^{2}-2 \sum m_{i} d_{i}^{2}-\frac{2}{3} K D>\frac{e(V)}{3} \text {. }
$$

We want to show that there is $K(V, \Delta)$ such it holds for $D=\sum m_{i} d_{i}$ satisfying $m_{i}>K(V, \Delta)$ for all $i$.

Let $d_{1} \in \Delta$ be class such that $d_{1}^{2}>0$. Let $K(V, \Delta)$ be the maximum of the real roots of following polynomials $f_{i}(m)$ or 1 where

and

$$
f_{1}(m)=m^{2} d_{1}^{2}-2 m d_{1}^{2}-\frac{2}{3} m K d_{1}-\frac{e(V)}{3}
$$

$$
f_{i}(m)=m^{2} d_{i}^{2}-2 m d_{i}^{2}-\frac{2}{3} m K d_{i}
$$

for each $i>1$ such that $d_{i}^{2}>0$.

Then for $D=\sum m_{i} d_{i}, m_{i}>K(V, \Delta)$ for each $i$ with $d_{i}^{2}>0$ one has $f_{i}\left(m_{i}\right)>0$ and using

$$
D^{2}=\sum_{i, d_{i}^{2}>0} m_{i}^{2} d_{i}^{2}+\sum_{i, d_{i}^{2}<0} m_{i}^{2} d_{i}^{2}+2 \sum_{i<j} m_{i} m_{j} d_{i} d_{j}
$$

one obtains:

$$
\begin{gathered}
D^{2}-2 \sum m_{i} d_{i}^{2}-\frac{2}{3} K D-\frac{e(V)}{3}= \\
\sum_{i, d_{i}^{2}>0} m_{i}^{2} d_{i}^{2}+2 \sum_{i, j} m_{i} m_{j} d_{i} d_{j}-2 \sum_{i, d_{i}^{2}>0} m_{i} d_{i}^{2}+\sum_{i, d_{i}^{2}<0}\left(m_{i}^{2}-2 m_{i}\right) d_{i}^{2} \\
-\frac{2}{3} \sum_{i, d_{i}^{2}>0} m_{i} K d_{i}-\frac{2}{3} \sum_{i, d_{i}^{2}<0} m_{i} K d_{i}-\frac{e(V)}{3} \geq
\end{gathered}
$$

(since there are no classes $d_{i}^{2}<0, K d_{i}>0$ by assumption)

$$
\begin{gathered}
\sum_{i, d_{i}^{2}>0} m_{i}^{2} d_{i}^{2}-2 \sum_{i, d_{i}^{2}>0} m_{i} d_{i}^{2}-\frac{2}{3} \sum_{i, d_{i}^{2}>0} m_{i} K d_{i}-\frac{2}{3} \sum_{j, d_{i}^{2}<0, K d_{i}<0} m_{i} K d_{i}-\frac{e(V)}{3} \geq \\
\sum_{i} f_{i}\left(m_{i}\right)>0 .
\end{gathered}
$$

The first inequality uses that $m_{i}=1$ for curves with $d_{i}^{2}<0$ as was pointed right after the inequality (4) and the positivity of other dropped terms. Therefore the inequalities (5) and (13) are satisfied for $\alpha=2$. The latter implies that a pencil of curves in $H^{0}(V, \mathcal{O}(D))$ has at most 11 reduced fibers with components having classes only in $\Delta$.

Finally we will show Corollary 1.3. 
Proof of Corollary 1.3. Parts $\mathrm{A}$ and $\mathrm{B}$ are an immediate consequence of Theorem 1.2. We will show that the fundamental groups of the complements to a union of $r+1$ members of a pencil have the form described in (C). Consider the map $\pi: V \backslash \mathcal{D} \rightarrow \mathbb{P}^{1} \backslash \mathcal{R}$, Card $\mathcal{R}=r+1$ corresponding to the pencil and let $\mathcal{R}=R \bigcup S$ where $R$ (resp. $S$ ) are the images of singular (resp. smooth) fibers of $\pi$. Let $D_{1} \subset \mathbb{P}^{1}$ be a disk containing all critical values of $\pi$ outside of $R$ and let $D_{2} \subset \mathbb{P}^{1}$ be a disk intersecting $D_{1}$ at one point and containing $\mathcal{R}$. Let $H=\pi_{1}\left(\pi^{-1}\left(D_{1}\right)\right)$. The fundamental group of $\left.\pi\right|_{\pi^{-1}\left(D_{2}\right)}$ is isomorphic to the extension (1) since over $D_{2}$ the map $\pi$ is a locally trivial fibration and $\pi_{2}\left(D_{2} \backslash \mathcal{R}\right)=0$. Finally $V \backslash \mathcal{D}$ can be retracted onto a union of the preimages of $D_{1}$ and $D_{2}$ and van Kampen theorem gives a presentation as the amalgamated product with $\Sigma=\pi^{-1}\left(D_{1} \cap D_{2}\right)$ i.e. the complement in a generic fiber, i.e. a closed Riemann surface, to the set of base points of the pencil which is the extension of $\pi$ to $\tilde{V}$. Since the set of isotopy classes of pencils in one of linear system $H^{0}(V, \mathcal{O}(\delta))$ is finite and for a fixed pencil each subgroup $H$ is determined by the subset $R$ of the total set of critical points of $S$ with reducible preimages, the finiteness claim follows.

\section{Pencils On $\mathbb{P}^{2}$}

In remaining two sections we will consider concrete examples of estimates of type of fibers of pencils on surfaces for various choices of the types $\Delta$ of components of reducible curves $\mathcal{D} \subset V$. In many cases the thresholds $M_{1}(V, \Delta), M_{2}(V, \Delta)$ can be made more explicit. In this section we consider the case $V=\mathbb{P}^{2}$.

4.1. General estimates. In the case $\Delta=[1] \subset \mathbb{Z}=\mathrm{NS}\left(\mathbb{P}^{2}\right)$ one has $M_{2}\left(\mathbb{P}^{2}, \Delta\right)=$ 3 (cf. [10, [13, ,6], 19]). Indeed, as was shown in these references, a pencil of curves can have at most 4 fibers which are unions of lines unless this is a pencil of lines. If $\mathcal{A}$ is an arrangement of lines such that one has an essential surjection $\pi_{1}\left(\mathbb{P}^{2} \backslash \mathcal{A}\right) \rightarrow F_{r}$, in the sense described in assumption $(* *)$ made in Preface, then the holomorphic map $\Phi$ described in the proof of the Theorem 1.2 has $r+1$ reducible fibers which union coincides with $\mathcal{A}$ (i.e. any line of $\mathcal{A}$ belongs to one of the these $r+1$ fibers of $\Phi$ ). If $r>3$ then the pencil must be a pencil of lines and $\mathbb{P}^{2} \backslash \mathcal{A}$ is fibered over $\mathbb{P}^{1}$ with $r+1$ points removed and fiber isomorphic to $\mathbb{C}$. Hence $\pi_{1}\left(\mathbb{P}^{2} \backslash \mathcal{A}\right)=F_{r}$.

There are pencils of curves of arbitrary large degrees $d$ containing arrangements of lines with 3 fibers which are union of lines (for example the curves $C_{d}$ given by equation $\lambda\left(x^{d}-y^{d}\right)+\mu\left(y^{d}-z^{d}\right)=0$ and hence finiteness of the number of pencils of curves for which a union of reducible fibers is a union of lines and admits surjection $\pi_{1}\left(\mathbb{P}^{2} \backslash C_{d}\right) \rightarrow F_{r}$ may take place only for $r>2$ i.e. $M_{1}\left(\mathbb{P}^{2}, \Delta\right) \geq 2$. There is only one known pencil of curves with 4 fibers which are unions of lines (pencil of cubics with union of reducible fibers being 12 lines containing 9 inflection points of a smooth cubic). Finitness of the number of pencils with 4 fibers being a unions of lines is equivalent to $M_{1}(\Delta)=2$.

Now consider the case $\Delta_{k}=\{[1], \ldots,[k]\} \subset \mathrm{NS}\left(\mathbb{P}^{2}\right)$. Theorem 1.2 yields the following:

Corollary 4.1. There exists a function $k \mapsto M_{k}=M_{2}\left(\mathbb{P}^{2}, \Delta_{k}\right) \in \mathbb{Z}_{+}$such that a curve $\mathcal{C}$, having the degrees of each of its irreducible components at most $k$ and such that there is surjection $\pi_{1}\left(\mathbb{P}^{2} \backslash \mathcal{C}\right) \rightarrow F_{r}$ where $r>M_{k}$, is a union $\mathcal{C}=$ $C_{1} \cup \ldots \cup C_{s}, s \geq r+1$ of possibly reducible curves $C_{i}, 1 \leq i \leq s$ which are members 
of a pencil of curves of degree at most $k$ (i.e. $\mathcal{C}$ is composed of curves of a pencil of degree at most $k$ ).

Corollary 3.3 shows that the pencils of curves of degree $d$ in $\mathbb{P}^{2}$ have independent of $d$ or $k$ bound on the number of fibers which are unions of irreducible curves of degree at most $k$ provided $d \gg k$. Proposition below makes it more explicit. It shows that the maximal number $\rho_{d, k}\left(\mathbb{P}^{2}\right)$ of reducible fibers with degrees of components at most $k$ (we call such pencils $k$-reducible) in a pencils of curves of degree $d \geq 2 k$ is at most 11 . Hence the maximal rank of a free quotient of the fundamental group of the complements to a union of members of a pencil of curves of degree greater than or equal to $2 k$, having the degrees of irreducible components not exceeding $k$, is $\rho_{d, k}\left(\mathbb{P}^{1}\right)-1$. Since this does not provide information about pencils of the curves of degree between $k$ and $2 k$ we have:

$$
M_{k}+1 \geq \rho_{d, k}\left(\mathbb{P}^{2}\right), \quad \rho_{d, k}\left(\mathbb{P}^{2}\right) \leq 11(d \geq 2 k) .
$$

Moreover, as follows from Corollary 4.3 , the constant 9 in the theorem 1.4 can be decreased: $M_{1}\left(\mathbb{P}^{2}, \Delta_{k}\right)<6$.

Proposition 4.2. Assuming $d=n k+d_{0} \geq 2 k, 0 \leq d_{0}<k$ (or equivalently $n \geq 2$ ), one has the following universal bounds

$$
\rho_{d, k}\left(\mathbb{P}^{2}\right) \leq \begin{cases}6 & \text { if } k=2, \\ 8 & \text { if } k=3 \\ 9 & \text { if } k=4,5 \\ 10 & \text { if } 6 \leq k \leq 11 \\ 11 & \text { otherwise. }\end{cases}
$$

In particular $K\left(\mathbb{P}^{2}, \Delta_{k}\right)=2$.

Proof. In a pencil of curves of degree $d=n k+d_{0}$, a reducible member with degrees of irreducible components being at most $k$ must have at least $n$ components of degree $k$ and one component of degree $d_{0}$ and hence in notations used in inequality (13) taking as $F_{j}$ a smooth curve of degree $d_{0}$ for one of these components and smooth curves of degree $k$ for $n$ remaning ones, one has $d^{2}-3 d+\sum e\left(F_{j}\right) \geq$ $d^{2}-3 d+n\left(3 k-k^{2}\right)+3 d_{0}-d_{0}^{2}=\left(d-d_{0}\right)\left(d+d_{0}-k\right)$. Therefore we obtain from (13):

$$
\rho_{d, k}\left(\mathbb{P}^{2}\right) \leq 2 \frac{e(V)+3 D^{2}+2 K D}{D^{2}+K D+\sum e\left(F_{j}\right)} \leq \frac{6(d-1)^{2}}{\left(d-d_{0}\right)\left(d+d_{0}-k\right)} .
$$

The condition that a constant $\alpha$ is an upper bound of the right hand term in 16 is equivalent to positivity of the function

$$
\begin{aligned}
h(k, n, \alpha) & =n k\left((n-1) k+2 d_{0}\right) \alpha-6\left(n k+d_{0}-1\right)^{2} \\
& =(\alpha-6) k^{2} n^{2}+\left(12+\alpha\left(2 d_{0}-k\right)\right) k n-6 .
\end{aligned}
$$

For $\alpha=12$ (and $n \geq 2$ ) one has:

$$
h(k, n, \alpha) \geq 6 \times 4 k^{2}+(12-12 k) k \times 2-6>0
$$

for $k \geq 1$. The rest of inequalities (15) follows by direct verification.

The second part of the Proposition follows from inequality (17) as well. 
Corollary 4.3. For the saturated set $\Delta_{k} \subset \mathrm{NS}\left(\mathbb{P}^{2}\right)$ one has

$$
\begin{gathered}
2 \leq M_{1}\left(\mathbb{P}^{2}, \Delta_{1}\right) \leq 3 \\
2 \leq M_{1}\left(\mathbb{P}^{2}, \Delta_{k}\right) \leq M_{1}\left(\mathbb{P}^{2}, \Delta_{k^{\prime}}\right)<6, \text { if } k \leq k^{\prime} .
\end{gathered}
$$

In other words, there is only a finite number of different pencils of curves with more than six $k$-reducible fibers. Alternatively, for $r>5$ there are only finitely many curves, with components of degree at most $k$ and not composed of a pencil, admitting a surjections $\pi_{1}\left(\mathbb{P}^{2} \backslash \mathcal{D}\right) \rightarrow F_{r}$.

Proof. Indeed for $\alpha>6$ and fixed $k$ the function $h(k, n, \alpha)$ in (17) takes only finitely many negative values.

Corollary 4.4. The maximal number of reducible curves $\rho_{d, d-1}\left(\mathbb{P}^{2}\right)$ in a primitive base-component-free pencil of degree $d$ is at most $3(d-1)$ and there exist pencil of curves of degree $d$ with $3(d-1)$ reducible fibers. In particular $M_{2}\left(\Delta_{k}\right) \geq 3 k-1$.

Proof. It is an immediate consequence of the bound (16):

$$
\rho_{d, k}\left(\mathbb{P}^{2}\right) \leq\left[\frac{3(d-1)^{2}}{e_{r e l, k}}\right] \leq\left[\frac{6\left(n k+d_{0}-1\right)^{2}}{n k\left((n-1) k+2 d_{0}\right)}\right],
$$

applied to the particular case $k=d-1$, that is, $n=1$ and $d_{0}=1$. The existence is a consequence of the example of a pencil due to Ruppert.

4.1.1. Ruppert's Example. For the sake of completeness we will briefly discuss the sharpness of the linear bound given in Corollary 4.4. Ruppert described in [14] a pencil of curves of any degree $d$ with exactly $3(d-1)$ reducible fibers. Consider the net $\mathcal{N}$ in $\mathbb{P}^{2}$ given by the following curves $\mathcal{C}_{\lambda}$ of degree $d$ defined by the equation:

$$
F_{\lambda}\left(x_{0}, x_{1}, x_{2}\right)=\lambda_{0} x_{0}\left(x_{1}^{d-1}-x_{2}^{d-1}\right)+\lambda_{1} x_{1}\left(x_{2}^{d-1}-x_{0}^{d-1}\right)+\lambda_{2} x_{2}\left(x_{0}^{d-1}-x_{1}^{d-1}\right)
$$

for any $\lambda=\left[\lambda_{0}: \lambda_{1}: \lambda_{2}\right] \in \mathbb{P}^{2}$. One has the following properties:

(1) The curves $\mathcal{C}_{[1: 0: 0]}, \mathcal{C}_{[0: 1: 0]}$, and $\mathcal{C}_{[0: 0: 1]}$ are products of $d$ lines $x_{i}\left(x_{j}^{d-1}-\right.$ $\left.x_{k}^{d-1}\right),\{i, j, k\}=\{0,1,2\}$.

(2) The generic member of $\mathcal{N}$ is smooth. In order to check this note that

$$
\begin{gathered}
\mathcal{C}_{[1: 0: 0]} \cap \mathcal{C}_{[0: 1: 0]} \cap \mathcal{C}_{[0: 0: 1]}= \\
\left\{P_{0}=[1: 0: 0], P_{1}=[0: 1: 0], P_{2}=[0: 0: 1], Q_{i, j}=\left[1: \zeta^{i}: \zeta^{j}\right]\right\},
\end{gathered}
$$

where $\zeta^{d-1}=1$ are the $(d-1)^{2}+3 \leq d^{2}$ base points of this net. By direct calculation of the Jacobian of $F_{\lambda}$, one can check that the base points are the only singular points of $\mathcal{C}_{\lambda}$ for a finite number of values of $\lambda$ and hence by Bertini's Theorem, the generic member is smooth.

(3) The curve $\mathcal{C}_{\lambda}$ is reducible if $\lambda$ satisfies $S(\lambda)=0$ where $S(\lambda)$ is the degree $3(d-1)$ polynomial

$$
S(\lambda)=\left(\lambda_{0}^{d-1}-\lambda_{1}^{d-1}\right)\left(\lambda_{1}^{d-1}-\lambda_{2}^{d-1}\right)\left(\lambda_{2}^{d-1}-\lambda_{0}^{d-1}\right) .
$$

In other words, the net $\mathcal{N}$ intersects the discriminant variety $\mathcal{D}$ in its locus of reducible curves and the intersection splits as a product of $3(d-1)$ lines.

(4) A pencil in $\mathcal{N}$ is given as $\mathcal{P}=\left\{\mathcal{C}_{\lambda} \in \mathcal{N} \mid L(\lambda)=0\right\}$, where $L$ is a linear form. If $L(\lambda)$ is in general position with respect to $S(\lambda)$, then $L(\lambda)$ defines a pencil with exactly $3(d-1)$ reducible fibers.

(5) Moreover, if $\mathcal{C}_{\lambda}$ is a generic point of $\mathcal{N} \cap \mathcal{D}$, then $\mathcal{C}_{\lambda}$ is the union of a line and a smooth curve of degree $(d-1)$. 


\subsection{Examples of pencils with a maximal number of members composed} of quadrics. Let

$$
\rho_{k}\left(\mathbb{P}^{2}\right):=\max \left\{\rho_{d, k}\left(\mathbb{P}^{2}\right) \mid d \geq 2 k\right\} .
$$

It follows from [10, 13 that $\rho_{1}\left(\mathbb{P}^{2}\right)=4$ and the arrangement of 12 lines containing 9 inflection points of a smooth cubic provides an example of a pencil with 4 fibers which are unions of lines. Our purpose in this section will be to study $\rho_{2}\left(\mathbb{P}^{2}\right)$.

4.2.1. The bound $\rho_{d, 2}$. By Corollary 4.4 we know that $\rho_{3,2}\left(\mathbb{P}^{2}\right)=6$ and $(15)$, which is applicable for the remaining $d$, shows that $\rho_{d, 2}\left(\mathbb{P}^{2}\right) \leq 6$ for all $d \geq 4$. It is the purpose of this section to make this into an equality by constructing a pencil of quartics with six quartics composed of quadrics.

Consider a pencil of conics $\Lambda$ in general position and three lines $L_{1}, L_{2}, L_{3}$ such that there exist three conics $C_{1}, C_{2}, C_{3} \in \Lambda$ such that $L_{i}$ is tangent to $C_{j}$ and $C_{k}$ with $\{i, j, k\}=\{1,2,3\}$. This can be achieved for instance with the pencil $\Lambda=\left\{\alpha\left(x^{2}-z^{2}\right)+\beta\left(y^{2}-z^{2}\right)\right\}$, the lines

$$
L_{1}=\sqrt{2} x+i \sqrt{2} y+\sqrt{3} z, \quad L_{2}=2 x+i y+\sqrt{3} z, \quad L_{3}=\sqrt{2} x+\sqrt{2} y-3 z
$$

and the conics

$$
C_{1}=x^{2}+2 y^{2}-3 z^{2}, \quad C_{2}=2 x^{2}+y^{2}-3 z^{2}, \quad C_{3}=2 x^{2}-y^{2}-z^{2} .
$$

Let $\kappa$ denote the Kummer cover of order two associated with the abelian $\mathbb{Z}_{2} \times \mathbb{Z}_{2^{-}}$ cover ramified along $L_{1}, L_{2}$, and $L_{3}$ (i.e. associated with surjection $\pi_{1}\left(\mathbb{P}^{2} \backslash \bigcup_{1}^{3} L_{i}\right) \rightarrow$ $\mathbb{Z}_{2}^{3} / \mathbb{Z}_{2}$ sending the meridian of $L_{i}$ to the $i$-th component of $\mathbb{Z}_{2}^{3}$; in appropriate coordinates it is $\mathbb{P}^{2} \rightarrow \mathbb{P}^{2}$ given by $\left.\left[x_{0}, x_{1}, x_{2}\right] \rightarrow\left[x_{0}^{2}, x_{1}^{2}, x_{2}^{2}\right]\right)$. Note that $\Lambda^{\prime}=$ $\kappa^{*}(\Lambda)$ becomes a pencil of quartics intersecting transversally at the 16 points in the preimage of the base points of $\Lambda$ and also that $\kappa^{*}\left(C_{i}\right), i=1,2,3$ is a union of two conics. Finally, note that $\Lambda$ contains 3 singular fibers $C_{i}^{\prime}, i=1,2,3$ which are products of two lines. Hence $\kappa^{*}\left(C_{i}^{\prime}\right)$ is also a product of two conics intersecting transversally.

Additivity of Euler characteristic or the main result of [7] allows to relate the Euler characteristic of the surface $V$, the Euler characteristic of the generic fiber $e_{\hat{\varphi}}\left(t_{0}\right)$ (a Riemann surface of genus $\left(\begin{array}{c}4-1 \\ 2\end{array}\right)=3$ ), and the relative Euler characteristic of the singular fibers $e_{\text {rel }}$ as follows:

$$
e(X)=3+|B|=3+16=e\left(\mathbb{P}^{1}\right) e_{\hat{\varphi}}\left(t_{0}\right)+6 e_{r e l}+n=2 \cdot(-4)+6 \cdot 4+n,
$$

where $n$ is the relative Euler characteristic of the remaining singular fibers. Hence $n=3$, which is the number of additional nodal quartics in the pencil $\Lambda^{\prime}$.

Proposition 4.5. The pencil $\Lambda^{\prime}$ above is a primitive base-component-free pencil of quartics with six members being unions of quadrics. Therefore $M_{2}+1=\rho_{2}\left(\mathbb{P}^{2}\right)=6$.

\section{Completely Reducible fibers of Pencils on SuRfaces in $\mathbb{P}^{3}$}

The purpose of the remaining section is to exhibit examples of pencils on surfaces with a large number of completely reducible divisors as well as bounds which follow from the calculations in section 3

We shall start with the case $V=\mathbb{P}^{1} \times \mathbb{P}^{1}, \Delta=\{(1,0),(0,1),(1,1)\}$. Consider as a lower bound for $\sum e\left(F_{j}\right)$ in 13 the case when a completely reducible fiber consists of $n$ smooth curves in the class $(1,1)$ and $m-n$ smooth curves in the class 
$(1,0)$. Then the sum of the Euler characteristic of its irreducible components $F_{j}$ satisfies $2 m \leq \sum e\left(F_{j}\right)$. Therefore

$2 \frac{e(V)+3 D^{2}+2 K D}{D^{2}+K D+\sum e\left(F_{j}\right)}=2 \frac{4+6 m n-4 m-4 n}{2 m n-2 m-2 n+\sum e\left(F_{j}\right)}<2 \frac{2+3 m n-2 m-2 n}{m n-n}<6$ i.e. the number of completely reducible fibers does not exceed 6 .

We will show that $M_{2}\left(\mathbb{P}^{1} \times \mathbb{P}^{1}, \Delta\right) \geq 3, M_{2}\left(V, \Delta_{1}\right)=4$ for smooth cubic surfaces and that $M_{2}(V, \Delta)$ can be arbitrarily large for general surfaces in $\mathbb{P}^{3}$ for appropriate saturated sets $\Delta$ on respective surfaces $\left(\Delta_{1}\right.$ is the set of classes of lines on a cubic surface).

5.1. Generalized Hesse arrangements on $\mathbb{P}^{1} \times \mathbb{P}^{1}$. The purpose of this section is to exhibit an example of a pencil of curves on $\mathbb{P}^{1} \times \mathbb{P}^{1}$ with 4 completely reducible fibers, showing that the Hesse pencil on $\mathbb{P}^{2}$ is not the only such pencil on a rational surface. We shall use geometric interpretation of the group law on cubic curve i.e. that selecting an inflection point as the zero, a triple of points adds up to zero if and only if the triple is collinear (cf. [18]).

5.1.1. A Special Configuration of Points. Consider 9 points on a smooth cubic $\mathcal{C} \subset \mathbb{P}^{2}$ satisfying Pascal's Theorem as in Figure 1 .

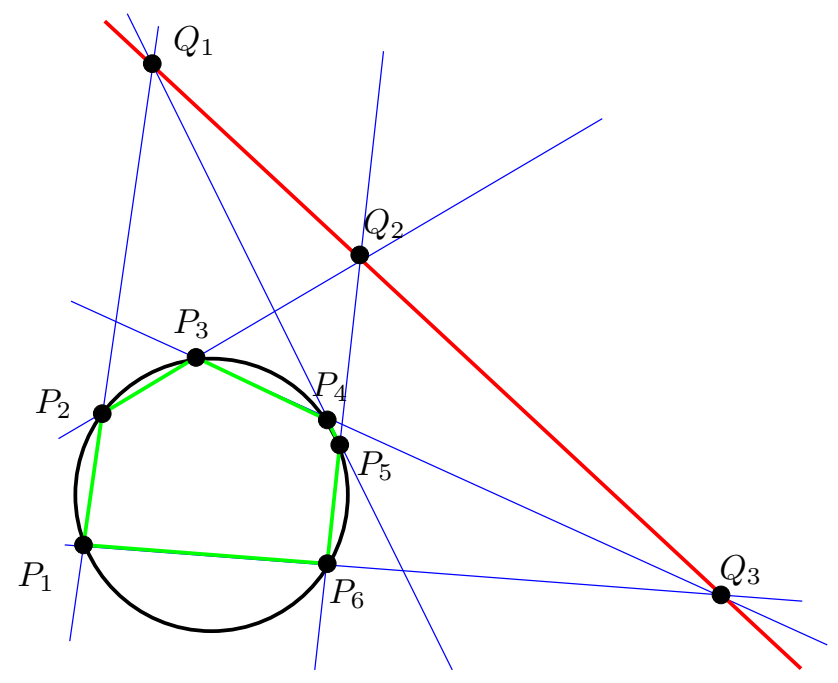

Figure 1. Pascal Point Configuration

Note that such a configuration of points would have to satisfy the following relations in the Picard group of the cubic:

$$
\begin{array}{ll}
P_{1}+P_{2}+Q_{1}=0, & P_{4}+P_{5}+Q_{1}=0 \\
P_{2}+P_{3}+Q_{2}=0, & P_{5}+P_{6}+Q_{2}=0, \\
P_{3}+P_{4}+Q_{3}=0, & P_{1}+P_{6}+Q_{3}=0 .
\end{array}
$$

In other words $P_{i}+P_{i+1}+Q_{j}=0, i \in \mathbb{Z}_{6}, j \in \mathbb{Z}_{3}$ and $\pi(i)=j$, where $\pi: \mathbb{Z}_{6} \rightarrow \mathbb{Z}_{3}$ is reduction modulo 3. By Pascal's Theorem

$$
\sum_{i} P_{i}=0, \quad \sum_{i} Q_{i}=0
$$


We also ask for three additional relations involving the diagonals:

$$
\begin{aligned}
& P_{1}+P_{4}+Q_{2}=0, \\
& P_{2}+P_{5}+Q_{3}=0, \\
& P_{3}+P_{6}+Q_{1}=0 .
\end{aligned}
$$

Definition 5.1. Any configuration of 9 points on a smooth cubic satisfying (19), (20), and 21) will be called a special Pascal configuration of points.

Lemma 5.2. If a six-tuple of points $P_{1}, . ., P_{6}$ forming complete intersection of conic and a smooth cubic $\mathcal{C}$ satisfies (19), (20) and (21), then $Q_{i}$ are collinear inflection points of $\mathcal{C}$. Vice versa, if $Q_{j}, j=1,2,3$ is triple of collinear inflection points and $P_{i} \neq Q_{j}$ is a point which order as a point of elliptic curve is non-equal to 2, then there exist 5 additional distinct points $P_{i^{\prime}}, i^{\prime} \neq i, 1 \leq i^{\prime} \leq 6$ such that relations (19), (20) and (21) are satisfied.

Proof. Let $P_{i}, Q_{j}$ be a collection of points satisfying (19), 21), 21). By symmetry, it is enough to show $3 Q_{1}=0$. Adding the relations in the first row of 190 and the last relation in 21) we obtain:

$$
P_{1}+P_{2}+P_{3}+P_{4}+P_{5}+P_{6}+3 Q_{1}=0
$$

and hence the first relation in 20 yields the claim.

Vice versa, again by symmetry it is enough to show that a choice of $P_{1}$ always determines remaining 5 points $P_{i}$ satisfying above relations. Since $P_{1}$ is not a point or order 2, it follows that the solution to the first relation in 19 satisfies $P_{2} \neq P_{1}$ and the order of $P_{2}$ is not equal to 2 . Hence the first 5 relations 19 allow to determine the points $P_{2}, . ., P_{6}$. Since $Q_{i}$ are collinear, we have the second relation (20) adding 3 relations not containing common $P_{i^{\prime}}$ among the 5 used to determine points $P_{i^{\prime}}$ this gives the first relation $(20)$ and hence the last relation $(19)$. Finally adding two relations in the first row of $\sqrt{19}$ and using $(20)$ we obtain the last relation (21) and the remaining relations (21) follow.

Proposition 5.3. For any smooth cubic $\mathfrak{C}$ there is a family of special Pascal configurations of points parametrized by a Zariski open subset of $\mathfrak{C}$.

Proof. It follows from the proof of previous Lemma since any choice of inflection points $Q_{i}, i=1,2,3, Q_{1}+Q_{2}+Q_{3}=0$ and $P, \operatorname{ord} P \neq 2$ determines uniquely a special Pascal configuration.

Generically, the three lines defined by (21) intersect in three double points, the conic defined in (20) is smooth, and intersects the line also defined in (20) transversally.

Consider the pencil of cubics generated by $\mathcal{C}_{1}:=\mathcal{L}_{12} \cup \mathcal{L}_{34} \cup \mathcal{L}_{56}$ and $\mathcal{C}_{2}:=$ $\mathcal{L}_{23} \cup \mathcal{L}_{45} \cup \mathcal{L}_{16}$, where $\mathcal{L}_{i j}$ is the line passing through $P_{i}$ and $P_{j}$. Note that the original smooth cubic $\mathcal{C}$ belongs to such a pencil and so do $\mathcal{C}_{3}:=\mathcal{L}_{14} \cup \mathcal{L}_{25} \cup \mathcal{L}_{36}$ and the union of the conic 2 passing through $P_{1}, \ldots, P_{6}$ and the line $\mathcal{L}$ passing through $Q_{1}, Q_{2}, Q_{3}$. In other words, one can find equations $C_{i}, Q, L$ of respective curves $\mathcal{C}_{i}, Q, i=1,2,3, \mathcal{L}$ such that $C_{3}=C_{1}-C_{2}$ and $Q L=C_{1}+C_{2}$.

Proposition 5.4. After blowing up the base points, the pencil of cubics described above induces an elliptic surface which is generically of type $I_{1}+I_{2}+3 I_{3}$.

Proof. The existence of $I_{2}$ and $3 I_{3}$ is given by hypothesis, then by a standard Euler characteristic computation, there should be an additional fiber of type $I_{1}$. 


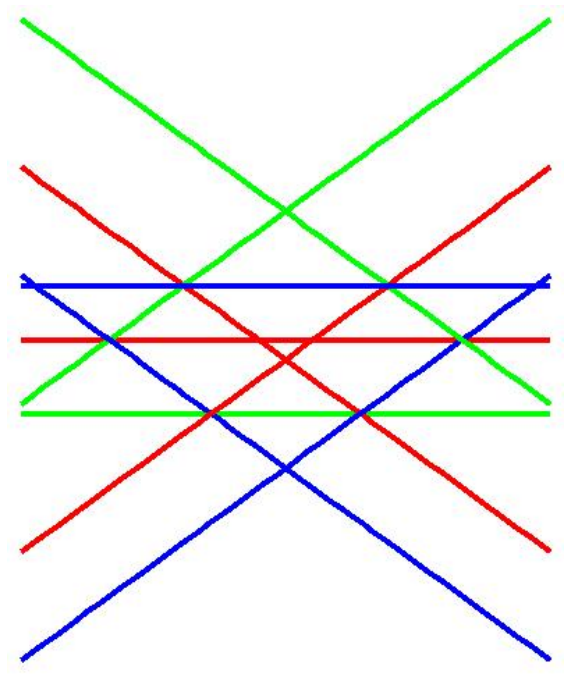

Figure 2. Special Pascal Point Configuration of type $I_{1}+I_{2}+3 I_{3}$

Example 5.5. Equations for Figure 2 can be given as:

$$
\begin{aligned}
& C_{1}=(2 y+(-2 \sqrt{3}+2) z)(\sqrt{3} x+y+\sqrt{3} z)(-\sqrt{3} x+y+\sqrt{3} z) \\
& C_{2}=(2 y+2 z)(\sqrt{3} x+y-\sqrt{3} z)(-\sqrt{3} x+y-\sqrt{3} z) \\
& C_{3}=(\sqrt{3} x-y-(2-\sqrt{3}) z)(\sqrt{3} x+y+(2-\sqrt{3}) z) y \\
& Q=3 x^{2}+3 y^{2}-3 z^{2}+2(2-\sqrt{3}) y z \\
& L=z
\end{aligned}
$$

Proposition 5.6. The types of the singular fibers of rational elliptic surfaces corresponding to the pencils given by the special Pascal configurations in Zariski open set in Proposition 5.3 which are not of generic type $I_{1}+I_{2}+3 I_{3}$ are as follows:

(1) $I_{2}+2 I_{3}+I V$

(2) $4 I_{3}$.

Proof. By hypothesis, we know that three singular fibers of a pencil of cubic curves forming a special Pascal configuration are unions of lines. Hence the types of the singular fibers of the corresponding rational elliptic surface are $I_{3}$ or $I V$, and since a fourth singular fiber of the pencil of cubics contains a line, the singular fiber of elliptic surface is of type $I_{2}, I_{3}, I I I$, or $I V$. Since the sum of Euler characteristics of the fibers is equal to 12 , there can only be three possibilities: $4 I_{3}, I_{2}+2 I_{3}+I V$, and $3 I_{3}+I I I$. A surface of type $4 I_{3}$ corresponds with the Hessian pencil, which comes from the choice of $P_{1}$ as an inflection point. A surface with configuration $I_{2}+2 I_{3}+I V$ appears when the three lines in $\mathcal{C}_{3}$ are concurrent. Finally, the surface with configuration $3 I_{3}+I I I$ does not exist according to Miranda's list of rational elliptic surfaces (cf.[12, p.197, item 92.]). 
Example 5.7. The special Pascal configuration of type $I_{2}+2 I_{3}+I V$ can be realized as the set of zeroes of:

$$
\begin{aligned}
& C_{1}=(2 y-\sqrt{3} z)(\sqrt{3} x+y+\sqrt{3} z)(-\sqrt{3} x+y+\sqrt{3} z) \\
& C_{2}=(2 y+\sqrt{3} z)(\sqrt{3} x+y-\sqrt{3} z)(-\sqrt{3} x+y-\sqrt{3} z) \\
& C_{3}=(3 x-\sqrt{3} y)(3 x+\sqrt{3} y) y \\
& Q=x^{2}+y^{2}-z^{2} \\
& L=z
\end{aligned}
$$

5.1.2. Generalized Hesse Arrangements on $\mathbb{P}^{1} \times \mathbb{P}^{1}$. Consider a double cover $\delta$ of $\mathbb{P}^{2}$ ramified along a smooth conic which is tangent to quadric $Q$ defined before Proposition 5.4 at 2 distinct points. The rational surface which realizes this covering is a ruled surface $\mathbb{P}^{1} \times \mathbb{P}^{1}$. Any irreducible component in the preimage of a line in $\mathbb{P}^{2}$ by $\delta$ has bidegree $(1,1),(1,0)$, or $(0,1)$ according to the relative position of the ramification locus and the line.

Definition 5.8. We say a curve in $\mathbb{P}^{1} \times \mathbb{P}^{1}$ is completely reducible if it is a union of irreducible components all being in the set $\Delta$ consisting of 3 classes: $(1,1),(1,0)$, or $(0,1)$.

Theorem 5.1. There exist pencils on $\mathbb{P}^{1} \times \mathbb{P}^{1}$ with four completely reducible fibers.

Proof. Consider a special Pascal configuration of type $I_{1}+I_{2}+3 I_{3}$ or $I_{2}+2 I_{3}+I V$ and a double cover $\delta$ of $\mathbb{P}^{2}$ ramified along a smooth conic, which is bitangent to $Q$. Then the pencil of cubics described above induces a pencil of curves of genus four on $\mathbb{P}^{1} \times \mathbb{P}^{1}$. The preimage of the $I_{2}$-fiber becomes two $(1,1)$-curves as preimage of the conic $Q$ and one more $(1,1)$-curve as a preimage of $\mathcal{L}$. The preimage of the $I_{3}$-fibers is a union of three $(1,1)$-curves.

Example 5.9. Consider the special Pascal configuration of type $I_{2}+2 I_{3}+I V$ provided in Example 5.7 and the covering $\delta: \mathbb{P}^{1} \times \mathbb{P}^{1} \rightarrow \mathbb{P}^{2}$ given by: $\delta([u, v],[s, t])=$ $[2(u t+v s), u s-v t, u s+v t]$, which ramifies along $\left\{x^{2}+4 y^{2}=4 z^{2}\right\}$ (a conic which is bitangent to $\left.\mathcal{Q}=\left\{x^{2}+y^{2}=z^{2}\right\}\right)$. Note that:

$$
\begin{aligned}
\delta^{*}\left(C_{1}\right)= & (6 u t+6 v s-(3+\sqrt{3}) u s-(3-\sqrt{3}) v t) \\
& (6 u t+6 v s+(3+\sqrt{3}) u s+(3-\sqrt{3}) v t)(v t-(7-4 \sqrt{3}) u s) \\
\delta^{*}\left(C_{2}\right)= & (6 u t+6 v s-(3-\sqrt{3}) u s-(3+\sqrt{3}) v t) \\
& (6 u t+6 v s+(3-\sqrt{3}) u s+(3+\sqrt{3}) v t)(v t-(7+4 \sqrt{3}) u s) \\
\delta^{*}\left(C_{3}\right)= & (6 u t+6 v s-\sqrt{3} u s+\sqrt{3} v t)(6 u t+6 v s+\sqrt{3} u s-\sqrt{3} v t)(u s-v t) \\
\delta^{*}(Q)= & (2 u t-(1-\sqrt{-3}) v s)(2 u t-(1+\sqrt{-3}) v s) \\
\delta^{*}(L)= & u s+v t
\end{aligned}
$$

Corollary 5.10. In notations of the Corollary 3.3 one has the bound $K(D=$ $(d, 1), \Delta,\left(\mathbb{P}^{1} \times \mathbb{P}^{1}\right) \geq K\left(D=(3,3), \Delta, \mathbb{P}^{1} \times \mathbb{P}^{1}\right) \geq 4$.

Moreover, primitive base-component-free pencils $\varphi: \mathbb{P}^{1} \times \mathbb{P}^{1} \rightarrow \mathbb{P}^{1}$ of bidegree $(3,3)$ and pencils confirming the equality $K\left((3,3), \Delta, \mathbb{P}^{1} \times \mathbb{P}^{1}\right)=4$ are not unique.

5.2. Completely reducible fibers on a cubic surface. Let $V$ be a smooth cubic surface in $\mathbb{P}^{3}$. The subsets $\Delta_{1} \subset \mathrm{NS}(V)$ consisting of the classes of 27 lines (generating the closure of the effective cone (cf. 4] p.485, section 9.1) is saturated.

Proposition 5.11. Let $V \subset \mathbb{P}^{3}$ be a smooth cubic as above, then $M_{2}\left(V, \Delta_{1}\right)=4$. 
Proof. The pencil of planes in $\mathbb{P}^{3}$ containing a fixed line induces a base point free pencil of residual for this line plane quadrics with 5 reducible fibers each consisting of 2 lines (cf. 4, section 9.1). On the other hand, inequality (13) or Corollary 1.5 . applied to divisor $D=H-L$ (here $H$ is the class of a hyperplane section and $L$ is the class of the fixed line) and using $D^{2}=0, K D=-2, e\left(F_{j}\right)=2, j=1,2, e(V)=9$, gives

$$
r+1 \leq 2 \frac{e(V)+3 D^{2}+2 K D}{D^{2}+K D+\sum e\left(F_{j}\right)}=5
$$

(summation over the irreducible components of a single member of the pencil). Therefore, $M_{2}\left(V, \Delta_{1}\right)=4$. For such $\Delta_{1}$, there are no pencils with members in a class $\delta \in \Delta_{1}$.

\subsection{Completely reducible fibers of pencils on surfaces of higher degree.}

Proposition 5.12. For any positive integer $d$ there is a surface $S_{d} \subset \mathbb{P}^{3}$ of degree $d$ and a pencil on it containing at least d completely reducible curves i.e. the curves with all irreducible components being lines.

Proof. The following is a well-known fact about construction of surfaces containing a large number of lines (cf. [15]). Consider $f(x, y)$ and $g(z, t)$ two homogeneous polynomials of degree $d$ with no multiple roots, then the surface

$$
S_{f, g}=\left\{[x: y: z: t] \in \mathbb{P}^{3} \mid f(x, y)=g(z, t)\right\}
$$

contains at least $d^{2}$ lines, namely all the lines $L_{i, j}, i, j=1, \ldots, d$ joining a point $P_{i}=\left[x_{i}: y_{i}: 0: 0\right]$ and a point $Q_{j}=\left[0: 0: z_{j}: t_{j}\right]$ where $f\left(x_{i}: y_{i}\right)=g\left(z_{j}, t_{j}\right)=0$.

The pencil of hyperplanes containing the line $L=\{x=y=0\}$ induces a pencil of curves on $S_{f, g}$. Given any point $P_{i}$, the hyperplane $H_{i}=\left\{y_{i} x=x_{i} y\right\}$ containing $P_{i}$ has to contain the lines $L_{i, 1}, \ldots, L_{i, d}$. Therefore this pencil contains at least $d$ completely reducible fibers.

\section{REFERENCES}

[1] D. Arapura, Geometry of cohomology support loci for local systems. I. J. Algebraic Geom. 6 (1997), no. 3, 563-597.

[2] E. Artal, J.I. Cogolludo-Agustín, and D. Matei, Characteristic varieties of quasi-projective manifolds and orbifolds, Geom. Topol. 17 (2013), 273-309.

[3] O. Debarre, Introduction to Mori theory. Universite Paris Diderot, 2016.

[4] I. Dolgachev, Classical algebraic geometry. A modern view. Cambridge University Press, Cambridge, 2012.

[5] J. Ellenberg, A. Venkatesh, The number of extensions of a number field with fixed degree and bounded discriminant. Ann. of Math. (2) 163 (2006), no. 2, 723-741.

[6] M. Falk, S. Yuzvinsky, Multinets, resonance varieties, and pencils of plane curves. Compos. Math. 143 (2007), no. 4, 1069-1088.

[7] B. Iversen, Critical points of an algebraic function. Invent. Math. 12 (1971), 210-224.

[8] J. Kollár, Rational curves on algebraic varieties. Ergebnisse der Mathematik und ihrer Grenzgebiete. 3. Folge. A Series of Modern Surveys in Mathematics, 32. Springer-Verlag, Berlin, 1996.

[9] R. Lazarsfeld, Positivity in algebraic geometry. I. Classical setting: line bundles and linear series. Ergebnisse der Mathematik und ihrer Grenzgebiete. 3. Folge. A Series of Modern Surveys in Mathematics, 48. Springer-Verlag, Berlin, 2004. 
[10] A. Libgober, S. Yuzvinsky, Cohomology of the Orlik-Solomon algebras and local systems. Compositio Math. 121 (2000), no. 3, 337-361.

[11] G. Malle, On the distribution of Galois groups. J. Number Theory 92 (2002), no. 2, 315-329.

[12] R. Miranda, Persson's list of singular fibers for a rational elliptic surface, Math. Z. 205 (1990), no. 2, 191-211.

[13] J.V. Pereira, S. Yuzvinsky, Completely reducible hypersurfaces in a pencil. Adv. Math. 219 (2008), no. 2, 672-688.

[14] W. Ruppert, Reduzibilität ebener Kurven, J. Reine Angew. Math. 369 (1986), 167-191.

[15] B. Segre, On arithmetic properties of quartic surfaces, Proc. London Math. Soc., (2) 49, 1947, 353-395.

[16] J.P. Serre, Groupes algébriques et corps de classes. Publications de l'institut de mathématique de l'université de Nancago, VII. Hermann, Paris 1959.

[17] I.Shafarevich et al, Algebraic Surfaces, Proc. Steklov Institute of Math. 75 (1965). Translated by American Mathematical Society. Rhode Island, 1967.

[18] J. Silverman, The arithmetic of elliptic curves. Second edition. Graduate Texts in Mathematics, 106. Springer, Dordrecht, 2009.

[19] S. Yuzvinsky, A new bound on the number of special fibers in a pencil of curves, Proc. Amer. Math. Soc. 137 (2009), no. 5, 1641-1648.

[20] O. Zariski, Algebraic surfaces. With appendices by S.S. Abhyankar, J. Lipman and D. Mumford. Preface to the appendices by Mumford. Reprint of the second (1971) edition. Classics in Mathematics. Springer-Verlag, Berlin, 1995.

Departamento de Matemáticas, iUma, Universidad de Zaragoza, C. Pedro Cerbuna 12, 50009 Zaragoza, Spain

Email address: jicogo@unizar.es

Department of Mathematics, University of Illinois, Chicago, IL 60607

Email address: libgober@uic.edu 